\title{
Reconstruction of the proximal humerus after extensive extraarticular resection for osteosarcoma: A report of two cases with clavicula pro humero reconstruction
}

\author{
YOSHIHIRO NISHIDA ${ }^{1}$, SATOSHI TSUKUSHI ${ }^{1}$, YOSHIHISA YAMADA ${ }^{3}$, YUZURU KAMEI ${ }^{2}$ \\ KAZUHIRO TORIYAMA ${ }^{2}$ and NAOKI ISHIGURO ${ }^{1}$
}

Departments of ${ }^{1}$ Orthopaedic Surgery, and ${ }^{2}$ Plastic Surgery, Nagoya University Graduate School of Medicine, 65-Tsurumai, Showa, Nagoya 466-8550; ${ }^{3}$ Department of Orthopaedic Surgery, Nagoya Memorial Hospital, 4-305, Hirabari, Tempaku, Nagoya 468-8520, Japan

Received April 18, 2008; Accepted June 20, 2008

\author{
DOI: 10.3892/or_00000116
}

\begin{abstract}
Two cases with clavicula pro humero reconstruction are described after extensive extraarticular wide resection of osteosarcoma due to the aggressive invasion, pre-operative pathological fracture, and relatively resistant to preoperative chemotherapy. Each patient had proximal humeral osteosarcoma and needed extraarticular resection. One necessitated more than two-third of humeral resection and reconstructed with rotated clavicle reconstruction with use of pasteurized intercalary autogenous bone graft. The other necessitated wide scapular resection, preserving acromioclavicular joint, could be reconstructed with rotated clavicle. Both had soft tissue reconstruction with pedicled lattissimus dorsi muscle flap for soft tissue defect. The Musculoskeletal Tumor Society score was $73 \%$ and $87 \%$, comparable for a previous report with clavicle pro humero reconstruction after conventional resection of proximal humerus or other reconstructive procedures. One had postoperative complication of prominent acromion with skin perforation. Both patients are alive with no evidence of disease, and no local recurrence. Clavicula pro humero reconstruction should be considered in selected patients for whom acromio-clavicular joint could be preserved after extensive resection of humerus or glenoid to obtain wide surgical margin.
\end{abstract}

\section{Introduction}

The proximal humerus is one of the primary sites of bone tumors and is the third most common site of origin for osteosarcoma $(1,2)$. Reconstruction after tumor resection of proximal humerus is occasionally challenging. Several procedures have been described for reconstruction of the

Correspondence to: Dr Yoshihiro Nishida, Department of Orthopaedic Surgery, Nagoya University Graduate School of Medicine, 65-Tsurumai, Showa, Nagoya, 466-8550, Japan

E-mail: ynishida@med.nagoya-u.ac.jp

Key words: proximal humerus, osteosarcoma proximal humerus. The reconstructive procedures of choice should be selected for each patient individually. An intensive invasion of primary malignant bone tumors arising in proximal humerus to surrounding soft tissues or intramedullary extension necessitates an extraarticular resection, or distal humerus resection. It results in an extensive bone deficiency, or an insufficiency of abductor mechanism. Clavicula pro humero reconstruction has been used after resection of not only proximal humerus (3) but also after humerus and glenoid resection (4) or of more humerus length followed by use of vascularized fibula (5). However, extent of glenoid resection was not detailed previously. Moreover, microsurgery for vascularized fibula is technically demanding and timeconsuming.

Two cases of clavicula pro humero reconstruction in patients with osteosarcoma (ages 13 and 19 years) showing aggressive invasion to surrounding soft tissues or intramedullary extension are described. Functional evaluation using the Musculoskeletal Tumor Society functional score and modified Toronto Extremity Salvage score, and complications in two patients are reported.

\section{Case report}

Case 1. A 13-year-old girl complained of pain in her right shoulder. She was seen at a nearby bone setter, and underwent physiotherapy for several weeks. Her pain was however increasing and she was seen by a local orthopaedist. A malignant bone tumor was suspected based on the plain radiographs, and she was referred to the Orthopaedic Oncology Unit at our hospital. Plain radiographs of the right shoulder revealed a permeative osteolytic lesion of the proximal humerus with periosteal reaction and pathological fracture (Fig. 1). T2-weighed magnetic resonance imaging (MRI) of the coronal view showed a heterogeneous changes in signal intensity from low to high in the humerus, extending to the central of diaphysis intramedullary, and invading the soft tissues extraskeletally. Other than increase of alkaline phosphatase, all laboratory examinations were all within normal limits. The specimens obtained by open biopsy through the anterior deltoid muscle approach revealed an 


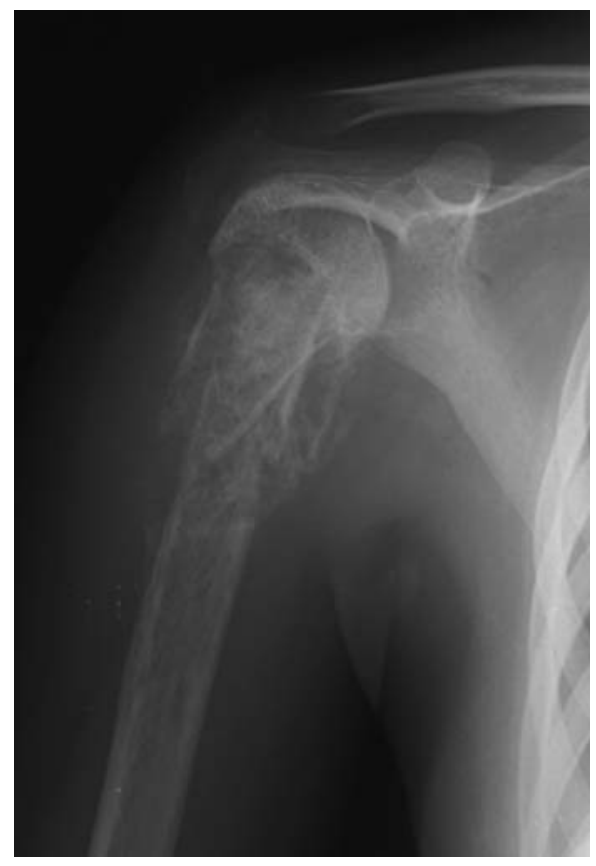

Figure 1. Case 1. An anteroposterior radiograph after preoperative chemotherapy.

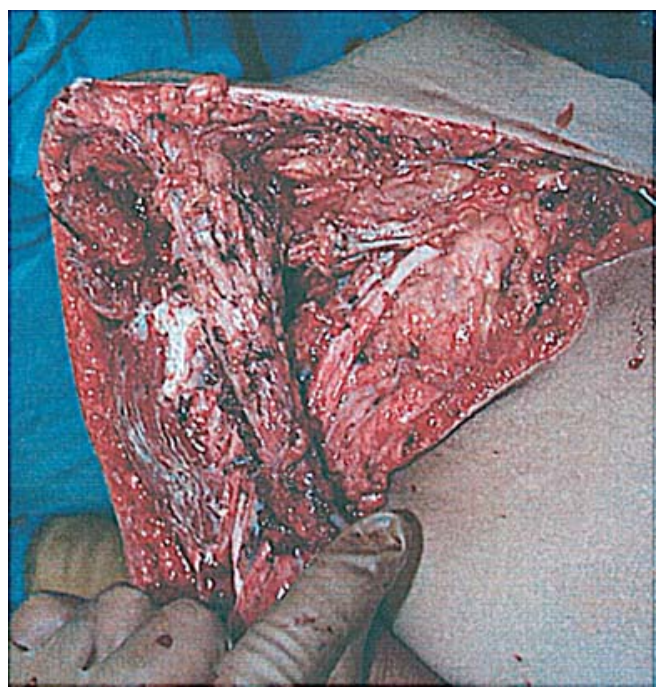

Figure 2. Case 1. Rotate the clavicle downwards preserving acromial branches of thoracoacromial vessels.

osteosarcoma. The patient was treated with 7 cycles of preoperative chemotherapy composed of cisplatin, methotrexate, doxorubicin, and ifosfamide. During the first 5 cycles of preoperative chemotherapy, the mass expanded more extraskeletally. However, in the latter 2 cycles, the size of mass stabilized, the osteosarcoma was subjected to surgical treatment. The operative settings were as following.

In a left lateral decubitus position, the right arm was prepared free. The surgical incision extended from the medial end of the clavicle, passed over coracoid process along the deltopectoral groove, and along the anteromedial aspect of the arm. A wide resection was performed, removing the site of biopsy in continuity with skin incision and the resected specimen. The majority of deltoid muscle, and part of

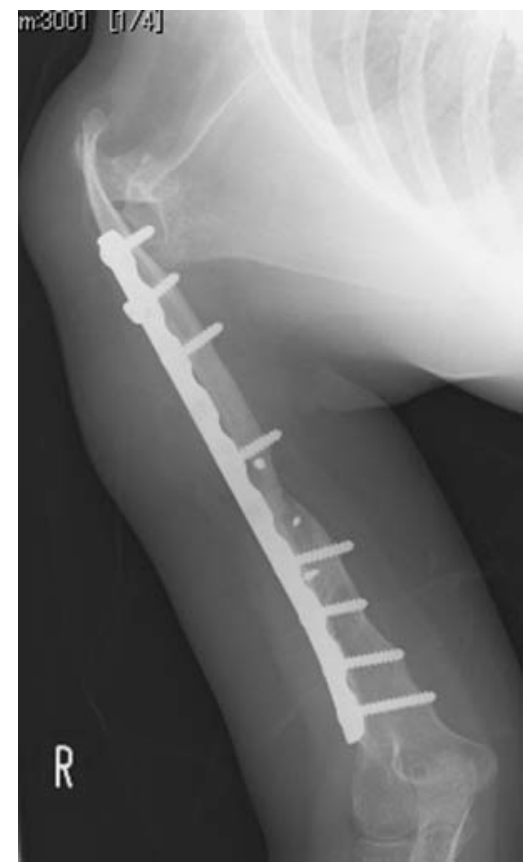

Figure 3. Case 1. A plain radiograph taken 6 months after surgery shows complete union of the rotated clavicle, pasteurized bone and humerus

brachialis, long head of biceps brachii, triceps brachii, and cuff muscles were resected in additon to the axillary nerve ligation. Nerves other than axillary nerve were preservevd. Extraarticular scapular osteotomy was done to obtain wide surgical margin from extraosseous tumor. A transverse osteotomy of the humeral shaft was made $3 \mathrm{~cm}$ distal to the distal intramedullary extent of the tumor, which was $5 \mathrm{~cm}$ proximal to the elbow joint. Third osteotomy was made at the clavicle, $1 \mathrm{~cm}$ lateral to the sterno-clavicular joint. Detaching trapezius, subscapular, and sternocleidomastoid muscles, the clavicle was clockwise-rotated downwards. During the procedure, the acromial branches of the thoracoacromial vessels were carefully preserved to maintain the nutrition of the rotated clavicle (Fig. 2), and the bleeding from the stump of the clavicle was confirmed. The length of the rotated clavicle available for reconstruction was $12.5 \mathrm{~cm}$ for $19 \mathrm{~cm}$ defect of humerus. Thus, $5 \mathrm{~cm}$ of resected humerus was pasteurized at $70^{\circ} \mathrm{C}$ for $15 \mathrm{~min}(6)$, and grafted intercalary between residual distal humerus and the rotated clavicle. The length of the used pasteurized bone was decided so to allow a $2-\mathrm{cm}$ shortning of the arm in order to prevent traction on the neurovascular structure, facilitate wound closure, and to maintain the function, such feeding, and washing face. An AO narrow dynamic compression plate was used for osteosynthesis extending from the rotated clavicle, over the intercalary pasteurized autogenous bone graft, to residual humerus. The remaining muscles were secured tight to the clavicle. Pedicled lattissimus dorsi muscle flap was used to cover the soft tissue defect.

Postoperatively, the upper limb was immobilized with a stockinette-Gilchrist bandage for 3 weeks, followed by an exercise of active and passive movement. Seven cycles of postoperative chemotherapy was performed after 3 weeks from the surgical treatment analogical to the preoperative chemotherapy. Plain radiographs at 6 months after operation 


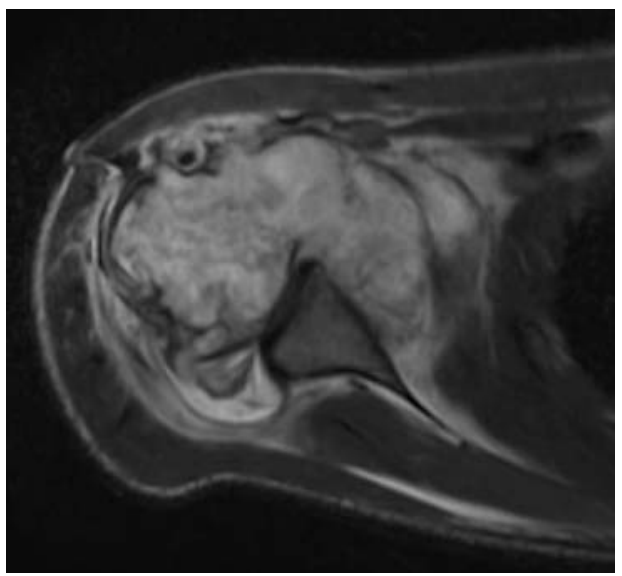

Figure 4. Case 2. A T2-weighed axial MRI scan shows the intraosseous and extraosseous extent to the anterior aspect of glenoid of the tumor.

revealed a bony union between not only the clavicle and the distal humerus (Fig. 3), but also the pasteurized bone and distal humerus. Bone scintigram with technetium-99mlabeled hydroxymethlenediphosphonate (HMDP) showed a good uptake in the pasteurized bone. No complication was observed postoperatively. The patient had a good function at 3 months after operation based on Musculoskeletal Tumor Society and International Symposium on Limb Salvage (7) evaluation with scores of 5 for pain, 4 for function, 4 for emotional acceptance, 4 for positioning of the hand, 5 for manual dexiterity, and 4 for lifting ability. The total score was $26(87 \%)$. Activities of daily living for upper extremity were also evaluated with Toronto Extremity Salvage score modified to Japanese lifestyle (3), and the score was 32 points $(80 \%)$. She could do recreational activities, such as beating a snare drum. Five months after operation, computed tomography revealed multiple lung metastasis. She recovered after a thoracotomy followed by high-dose chemotherapy with autologous peripheral blood stem cell transplantation based on carboplatin, etoposide, and melphalan. There was no evidence of disease at 3 years and 10 months after initial operation.

Case 2. Nineteen-year-old man complained of right shoulder pain, and was seen at nearby general hospital. Right proximal humeral lesion was suspected as a bone neoplasm based on MRI examination. He was presented to the Orthopaedic Oncology Unit at our hospital. Radiographs at presentation demonstrated an osteolytic lesion at medial aspect of right humeral neck with monolayered periosteal reaction. Including alkaline phosphatase, all laboratory examinations were all within normal limits. A needle biopsy under control of CT imaging failed to diagnose the lesion. Open biopsy was performed. The patient was diagnosed with an osteosarcoma of the right proximal humerus. Despite 6 cycles of preoperative chemotherapy based on cisplatin, methotrexate, ifosfamide, and doxorubicin, the tumor expanded extraskeletally (Fig. 4), and patient suffered from a proximal humerus pathological fracture. Two more cycles of preoperative chemotherapy composed of ifosfamide, carboplatin, and etoposide managed to stabilize the size of the tumor, and subsequently he was treated surgically.
A

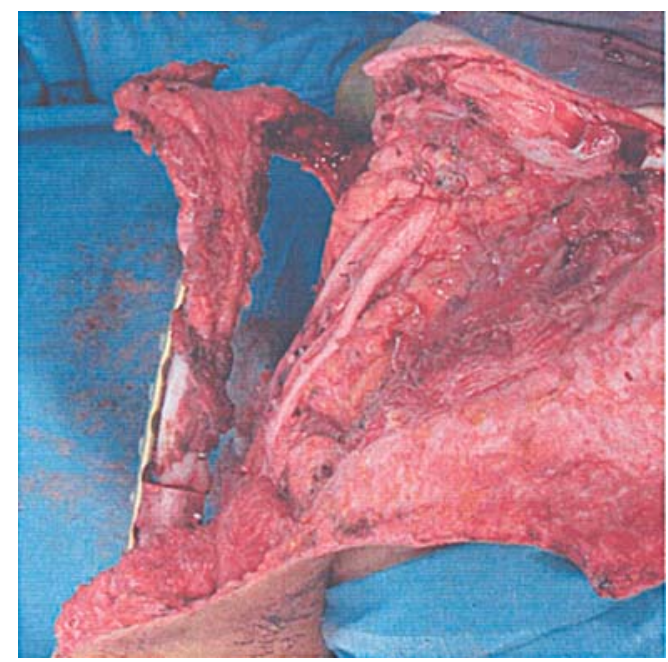

B

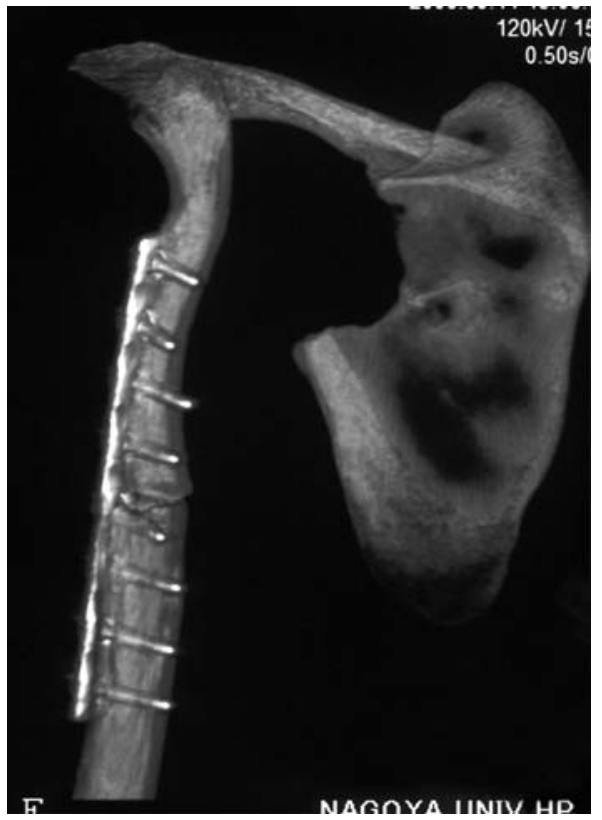

Figure 5. Case 2. (A) Rotated clavicle is fixed with distal humerus using a titanium plate, preserving acromion and scapular spine. (B) Three-dimensional CT imaging shows the exact resection margin of the scapula.

In a left lateral decubitus position, the right arm was prepared free. The surgical incision was similar as in case 1 . It extended from the medial end of the clavicle to the anteromedial aspect of the arm. A wide resection was performed, removing the site of biopsy. The extraosseous tumor expansion made the wide surgical margin difficult. It was indispensable to resect antero-lateral part of deltoid, part of cuff muscles, axillary nerve in addition to the ligation of posterior circumflex humeral vessels. Extending the skin incision posteriorly, posterior part of deltoid, infraspinatus, insertion of teres minor, teres major, long head and lateral head of triceps brachii muscles were excised. To preserve the scapular spine and perform an extraarticular resection, an reversed L-shaped osteotomy was made through this area with a chisel (Fig. 5). The humeral osteotomy was made at $15 \mathrm{~cm}$ distal from proximal end of humerus. Following the extraarticular resection, a clavicle osteotomy was made at $15 \mathrm{~cm}$ from acromio-clavicular joint. The clavicle was clock-wise rotated downwards, and osteosynthesized with the 
residual distal humerus by an 8-hole titanium plate (Fig. 5). Autologous bone tips obtained from scapula or clavicle were grafted to the junction of the rotated clavicle and the humerus. During this procedure, acromial branches of thoracoacromial vessels and thoracodorsal vessels had to be ligated to obtain wide surgical margin. Although the thoracoacromial vessels were ligated, bleeding from the stump of the rotated clavicle was confirmed, suggesting sufficient supply from the acromial branches of suprascapular vessels to the rotated clavicle. Due to the sacrifice of thoracodorsal vessels, soft tissue defect was covered with free vascularized lattissimus dorsi flap.

Postoperatively, the upper limb was immobilized for 3 weeks, followed by an exercise of active and passive movement. After first cycle of postoperative chemotherapy composed of ifosfamide, carboplatin, and etoposide, severe thrombocytopenia occurred, and diagnosed as an idio-pathic thrombocytopenic purpura (ITP). Rest of postoperative chemotherapy was abandoned, and high-dose steroid therapy followed by splenectomy led to the complete thrombocytopenia recovery. Transient radial nerve palsy occurred postoperatively, however, it recovered completely 4 months later. Because abundant amount of soft tissue around the acromioclavicular joint was resected to obtain wide surgical margin, free lattissimus dorsi muscle flap was not sufficient to cover the defect. Two weeks after operation, a prominence of acromio-clavicular joint with skin perforation occurred, which was not cured with conservative treatment. Curettage of acromio-clavicular joint and free rectus abdominus muscle flap at 4 months after initial surgery cured the skin perforation. Although there was no bony union obtained at 1 year and 6 months after surgery, bone scintigram revealed adequate uptake in the rotated clavicle. Despite of the inferior instability of acromio-clavicular joint, the patient had a good function of upper extremity with scores of 5 for pain, 2 for function, 3 for emotional acceptance, 3 for positioning of the hand, 5 for manual dexiterity, and 4 for lifting ability. Total score was $22(73 \%)$. A score of modified Toronto Extremity Salvage score was 29 points $(73 \%)$. He had no recurrence of the tumor at 1 year and 6 months after surgery.

\section{Discussion}

Reconstructive procedures after wide resection of proximal humerus include arthrodesis $(8,9)$, prosthesis $(8,10,11)$, osteoarticular allografts $(8,12,13)$, a free vascularized fibular graft $(14)$, and clavicula pro humero reconstruction $(3,4)$. The procedure should be carefully chosen depending on the extent of extraosseous tumor invasion, demand of the patients, and outcomes of the procedure itself including various kinds of complications. Whether extraarticular resection or intraarticular resection is suitable for osteosarcoma arising in proximal humerus has been contaversial. Although a rate of local recurrence seems to be less frequent in cases of extraarticular resection (7\%) than in those of intraarticular resection (18\%) (11), the necessity of resection of axillary nerve and part of abductor mechanisms may reduce the postoperative function in cases with extraarticular resection. In the current cases, choice of extraarticular resection was decided based on the resistant biological behavior of tumors to preoperative chemotherapy, and the extent of extraosseous tumor invasion. Axillary nerve and abductor mechanisms in addition to the cuff muscles had to be resected together with the tumor, what made not only proximal humerus but also soft tissue reconstruction difficult.

Clavicula pro humero reconstruction may be utilized if acromio-clavicular joint is preserved after wide resection of the tumor. A previous report described this procedure for the patients with malignant bone tumors of proximal humerus, however, most of the cases were reconstructed after intra-articular resection (3). Although another report of this procedure included cases of extraarticular resection, it did not describe the details concerning the extent of resection. In case 2 of our report, because most of musculature around the shoulder joint was resected and only acromio-clavicular joint and scapular spine were preserved after wide resection. Therefore a reconstruction with prosthesis would be inadequate to stabilize the shoulder, on the other hand, an arthrodesis or free vascularized fibular graft would be timeconsuming and technically demanding.

Acromial branches of thoracoacromial artery and suprascapular artery are considered to be important nourishing arteries to the rotated clavicle. In case 2, branches of thoracoacromial vessels were ligated and incised to obtain wide surgical margin. After sacrifice of these vessels, bleeding from the stump of rotated clavicle was well observed, suggesting the nutrition from branches of suprascapular artery was sufficient for the rotated clavicle. This fact might enlarge the indication of clavicula pro humero procedure in cases with extensive wide resecion. However, a complication after surgery, the perforation of acromio-clavicular joint through the skin, which necessitated curettage of the joint, damaged the artery from suprascapular vessels, may perturb the longterm evaluation for the nutrition of rotated clavicle.

Shortening of reconstructed proximal humerus is sometimes beneficial for elbow and wrist function, such as bringing food to mouth, drinking from a glass, however, an excess reduction causes cosmetic problems. Clavicula pro humero procedure, which was originally described by Sulamaa (15), has the capacity of longitudinal growth of the clavicula. In case 1 , there was $7 \mathrm{~cm}$ discrepancy in length between the rotated clavicle and resected proximal humerus, leading to cosmetic problems. Although a previous report described that a free vascularized fibular intercalary graft had a good clinical outcome (5), it needed microsurgery. In the current report, $5 \mathrm{~cm}$ of pasteurized intercalary bone graft was utilized to reduce the limb length discrepancy. Considering the early bone union, and good uptake by bone scintigram to the pasteurized bone, this technically relatively easy, and rapid procedure seems adequate.

Postoperative function of upper extremity for clavicula pro humero was comparable to other reconstructive procedures $(8,13,14,16)$ evaluated with MSTS scoring system. Although more bone and soft tissues were resected in current cases due to the extension of the tumor, the MSTS functional score was not reduced compared with a previous report using clavicula pro humero after intraarticular resection (3). It suggests that the stable postoperative function is predictable with clavicula pro humero reconstruction whether resection is intraarticular or not. 
The complication of this procedure in the current case was the prominence of the acromio-clavicular joint with skin perforation, which needed additional surgical treatment. An ample coverage with muscular flap to acromio-clavicular joint appears crucial for this procedure. Given that there was generally less complications with this procedure (15), clavicula pro humero reconstruction provides an adequate reconstruction for cases with extensive defect after wide resection of proximal humerus.

\section{References}

1. Glasser DB, Lane JM, Huvos AG, Marcove RC and Rosen G: Survival, prognosis, and therapeutic response in osteogenic sarcoma. The Memorial Hospital experience. Cancer 69: 698-708, 1992.

2. Marcove RC, Miké V, Hajek JV, Levin AG and Hutter RV: Osteogenic sarcoma under the age of twenty-one. A review of one hundred and forty-five operative cases. J Bone Joint Surg Am 52A: 411-423, 1970

3. Tsukushi S, Nishida Y, Takahashi M and Ishiguro N: Clavicula pro humero reconstruction after wide resection of the proximal humerus. Clin Orthop Relat Res 447: 132-137, 2006.

4. Rödl RW, Gosheger G, Gebert C, Lindner N, Ozaki T and Winkelmann W: Reconstruction of the proximal humerus after wide resection of tumours. J Bone Joint Surg Br 84B: 1004-1008, 2002.

5. Ozaki T, Hashizume H, Kunisada T, Kawai A, Nishida K, Sugihara $\mathrm{S}$ and Inoue $\mathrm{H}$ : Reconstruction of the proximal humerus with the clavicle after tumor resection: a case report. Clin Orthop Relat Res 385: 170-175, 2001.

6. Sugiura H, Yamamura S, Sato K, Katagiri H, Nishida Y, Nakashima $\mathrm{H}$ and Yamada $\mathrm{Y}$ : Remodelling and healing process of moderately heat-treated bone grafts after wide resection of bone and soft-tissue tumors. Arch Orthop Trauma Surg 123: 514-520, 2003
7. Enneking WF, Dunham W, Gebhardt MC, Malawar M and Pritchard DJ: A system for the functional evaluation of reconstructive procedures after surgical treatment of tumors of the musculoskeletal system. Clin Orthop Relat Res 286: 241-246, 1993.

8. O'Connor MI, Sim FH and Chao EY: Limb salvage for neoplasms of the shoulder girdle. Intermediate reconstructive and functional results. J Bone Joint Surg Am 78A: 1872-1888, 1996.

9. Rose PS, Shin AY, Bishop AT, Moran SL and Sim FH: Vascularized free fibula transfer for oncologic reconstruction of the humerus. Clin Orthop Relat Res 438: 80-84, 2005.

10. Kumar D, Grimer RJ, Abudu A, Carter SR and Tillman RM: Endoprosthetic replacement of the proximal humerus: long-term results. J Bone Joint Surg Br 85B: 717-722, 2003

11. Wittig JC, Bickels J, Kellar-Graney KL, Kim FH and Malawer MM: Osteosarcoma of the proximal humerus: longterm results with limb-sparing surgery. Clin Orthop Relat Res 397: 156-176, 2002.

12. Gebhardt MC, Roth YF and Mankin HJ: Osteoarticular allografts for reconstruction in the proximal part of the humerus after excision of a musculoskeletal tumor. J Bone Joint Surg Am 72A: 334-345, 1990 .

13. Getty PJ and Peabody TD: Complications and functional outcomes of reconstruction with an osteoarticular allograft after intra-articular resection of the proximal aspect of the humerus. J Bone Joint Surg Am 81A: 1138-1146, 1999.

14. Wada T, Usui M, Isu K, Yamawaki S and Ishii S: Reconstruction and limb salvage after resection for malignant bone tumour of the proximal humerus: a sling procedure using a free vascularised fibular graft. J Bone Joint Surg Br 81B: 808-813, 1999.

15. Sulamaa M: Upper extremity phocomelia. A contribution to its operative treatment. Clin Pediatr 2: 251-257, 1963.

16. Fuchs B, O'Connor MI, Padgett DJ, Kaufman KR and Sim FH: Arthrodesis of the shoulder after tumor resection. Clin Orthop Relat Res 436: 202-207, 2005. 